Pacific Journal of Mathematics

BOOLEAN POWERS, RECURSIVE MODELS, AND THE HORN 


\title{
BOOLEAN POWERS, RECURSIVE MODELS, AND THE HORN THEORY OF A STRUCTURE
}

\author{
G. C. NeLsoN
}

\begin{abstract}
This paper concerns the algebraic constructions of Boolean powers and bounded Boolean powers of structures $\mathfrak{U}$ for an arbitrary first-order language. The notion of $B$-separating is used to improve results about the logic of reduced power structures. For $\mathfrak{A}$ recursive we construct recursive models of the theory of each reduced power of $\mathfrak{A}$. Finally, it is shown that any complete theory is equivalent to a finitely axiomatizable extension of its Horn consequences.
\end{abstract}

0. Introduction and notation. Throughout this paper we have tried to conform to the terminology and notation described in [6]. However, there are a few exceptions which we will point out here. First, $L$ always denotes a first-order language with equality. A structure $\mathfrak{A}$ for $L$ is defined as usual and its domain is denoted by $|\mathfrak{A}|$ or $A$; the interpretation of a relation symbol $R$ of $L$ in $\mathfrak{A}$ is denoted by $R^{\mathfrak{A}}$ and the interpretation of a function symbol $f$ of $L$ in $\mathfrak{A}$ is denoted by $f^{\mathfrak{A}}$. By $\operatorname{Th}(\mathfrak{A})$ we mean the set of sentences of $L$ true in $\mathfrak{A}$. The symbol $\omega$ usually denotes the set of natural numbers and whenever we refer to an operation such as + or $\leq$ (order) on $\omega$, it is to be interpreted as the standard addition or order on $\omega$. By $\mathfrak{B}$ we always denote a nontrivial Boolean algebra with the first-order language $L=\left\langle\cap, \cup,{ }^{\prime}, 0,1\right\rangle$ and abuse our notation to always write $\cap$ for $\cap^{\mathscr{B}}$, etc. By 2 or $\{0,1\}$ we mean the Boolean algebra with two elements where 0 is the smallest element. Our notation for the elementary characteristic $\langle n, p, q\rangle$ of a Boolean algebra $\mathfrak{B}$ is defined in [7] or [29] except that we use $\omega$ instead of $\infty$.

In $\S 1$ we study the Boolean powers and bounded Boolean powers of structures $\mathfrak{U}$ for an arbitrary first-order language $L$. This idea was introduced by Burris in [3] for algebraic languages (first-order with only function symbols). By characterizing the elementary characteristics of complete Boolean algebras, we show that the theories of bounded Boolean powers are much more general than those of Boolean powers and partially answer a question of [3] by exhibiting a sentence preserved by all Boolean powers which is not equivalent to a disjunction of Horn sentences. We also introduce a standard language construction which allows one to 
obtain $B$-separating structures $\mathfrak{U}_{f}$ with algebraic language $L_{f}$ from $B$-separating structures $\mathfrak{A}$ with first-order language $L$. Moreover, this allows us to easily adapt many of the results of Burris [3] to our setting.

In $\$ 2$ we give a result promised in [22], namely, an explicit algebraic construction which yields recursive models of the theory of $\mathfrak{A}^{I} / D$ whenever $\mathfrak{A}$ is recursive.

In $\S 3$ we show that the results in [20] (\$3) hold for any first-order language $L$ with a binary (or higher order) relation symbol.

In $\S 4$ we study $\mathcal{H}(\mathfrak{U})$, the set of Horn structures true in $\mathfrak{A}$. We show that for $\mathfrak{A} T$-separating that $\mathfrak{U}^{I} / D$ is finitely axiomatizable over $\mathcal{H}(\mathfrak{U})$ iff $2^{I} / D$ is finitely axiomatizable. Also, we demonstrate that for all $\mathfrak{A}$ there is a sentence $\varphi$ such that $\mathcal{H}(\mathfrak{A}) \cup\{\varphi\}$ axiomatizes $\operatorname{Th}(\mathfrak{U})$.

1. Boolean powers. In this paper our main objects of study are the Boolean powers and bounded Boolean powers as defined in [3]. In order to generalize these definitions to an aribtrary first-order language and for the reader's convenience we state explicitly these definitions.

DeFinition 1.1. Let $\mathfrak{A}$ be a structure for a first-order language $L$ and $\mathfrak{B}=\left\langle B, \cap, \cup,^{\prime}, 0,1\right\rangle$ be a Boolean algebra. For $\mathfrak{A}$ infinite, $\mathfrak{B}$ is required to be complete. We denote by $\mathfrak{A}[\mathfrak{B}]$ the Boolean power of $\mathfrak{A}$ with respect to $\mathfrak{B}$ which is a structure for $L$ defined as follows: The domain $|\mathfrak{A}[\mathfrak{B}]|$ of $\mathfrak{A}[\mathfrak{B}]$ consists of all functions $\xi$ in ${ }^{A} B$ such that for all $a_{0}, a_{1}$ in $A, a_{0} \neq a_{1}$ implies $\xi\left(a_{0}\right) \cap \xi\left(a_{1}\right)=0$, and $\cup_{a \in A} \xi(a)=1$. Let $c$ be a constant symbol of $L, c^{\mathfrak{A}[\mathfrak{B}]}=\xi_{c}$ where $\xi_{c}\left(c^{\mathfrak{A}}\right)=1$ and $\xi_{c}(a)=0$, if $a \neq c^{\mathfrak{A}}$. Let $R$ be an $n$-ary relation symbol of $L$. Then $\left(\xi_{1}, \ldots, \xi_{n}\right) \in R^{\mathfrak{A}[\mathfrak{B}]}$, if for all $a_{1}, \ldots, a_{n}$ in $A, \xi_{1}\left(a_{1}\right) \cap \cdots \cap \xi_{n}\left(a_{n}\right) \neq 0$ implies $\left(a_{1}, \ldots, a_{n}\right) \in R^{\mathfrak{A}}$. Let $f$ be an $n$-ary function symbol of $L$, then

$$
f^{\mathfrak{A}[\mathfrak{B}]}\left(\xi_{1}, \ldots, \xi_{n}\right)(a)=\bigcup_{f^{\mathscr{N}}\left(a_{1}, \ldots, a_{n}\right)=a}\left(\xi_{1}\left(a_{1}\right) \cap \cdots \cap \xi_{n}\left(a_{n}\right)\right) .
$$

The bounded Boolean power $\mathfrak{A}[\mathfrak{B}]^{*}$ is defined for any Boolean algebra $\mathfrak{B}$, not necessarily complete, exactly as above except that the domain $\left|\mathfrak{A}[\mathfrak{B}]^{*}\right|$ of $\mathfrak{A}[\mathfrak{B}]^{*}$ consists of all functions $\xi$ in ${ }^{A} B$ such that $\{a \mid a \in A$ and $\xi(a) \neq 0\}$ is finite, $\cup_{a \in A} \xi(a)=1$, and $a_{0} \neq a_{1}$ implies $\xi\left(a_{0}\right) \cap \xi\left(a_{1}\right)=$ 0 .

Waszkiewicz and Weglorz in [30] construct for any reduced power $\mathfrak{A}^{I} / D$ an extremely elegant elementary substructure. We point out here 
that their models are isomorphic to some bounded Boolean power of $\mathfrak{A}$ and that this observation is implicit in [3] (Lemma 4.1). Given an arbitrary Boolean algebra $\mathfrak{B}$, let $I$ be the set of points of the Stone space of $\mathfrak{B}$ with its usual topology [13] or [27]. A subset of $I$ is clopen, if it is both open and closed. Let $\theta$ be the canonical Boolean isomorphism of the clopen subsets of $I$ onto $\mathfrak{B}$. As in [30] define $\mathfrak{U}^{\mathfrak{B} *}$ to be the substructure of $\mathfrak{U}^{I}=\prod_{l \in I} \mathfrak{A}$ with domain equal to the set of all functions $f$ in ${ }^{I} A$ such that range of $f$ is finite and for all $a$ in $A, f^{-1}(\{a\})$ is clopen. It is straightforward to verify that $\mathfrak{U}^{\mathfrak{B} *} \cong \mathfrak{A}[\mathfrak{B}]^{*}$ by $\sigma$ where $\sigma(f)=\xi$ such that for $a$ in $A, \xi(a)=\theta\left(f^{-1}(\{a\})\right)$. Alternatively, as in [32], $\left|\mathfrak{A}^{3 \mathcal{B}^{*}}\right|$ can be described as the set of all continuous functions from $I$ into $A$ where $A$ has the discrete topology since by the compactness of the Stone space such a continuous function has finite range.

From the above it is clear that the Feferman-Vaught-Weinstein Theorem holds for $\mathfrak{A}[\mathfrak{B}]^{*}$, see [5] which adapts the particular form of this result given in [6] (pp. 341-345) and observe that this gives a uniform setting in regards to [20].

It is important to recall that for the reduced power of $\mathfrak{A}, \mathfrak{A}^{I} / D \equiv$ $\mathfrak{A}\left[2^{I} / D\right]^{*}[3](T h .4 .3(\mathrm{v}))$ and that $\operatorname{Th}\left(\mathfrak{H}^{I} / D\right)$ is completely determined by $\operatorname{Th}(\mathfrak{H})$ and $\operatorname{Th}\left(2^{I} / D\right)$ [6] (Theorem 6.3.4). Ershov [7] ([6] Lemma 6.3.21) has shown that for any Boolean algebra $\mathfrak{B}$ there is a filter $D$ on $\omega$ such that $2^{\omega} / D \equiv \mathfrak{B}$. Consequently, when dealing with the theory of a reduced power of $\mathfrak{U}$ we frequently describe it by $\mathfrak{U}[\mathfrak{B}]^{*}$.

The complete theory of a Boolean algebra $\mathfrak{B}$ has been uniquely characterized by its elementary characteristic which we write as $\langle n, p, q\rangle$ and one should see [7], [29], [6], or [23] (but the notation is different in [6] and [23]). As in [24] or [23] we define $\langle n, p, q\rangle \times\langle r, s, t\rangle=$ the elementary characteristic of $\left(2^{I} / D\right)^{I^{\prime}} / D^{\prime}$ where $2^{I} / D \vDash\langle r, s, t\rangle$ and $2^{I^{\prime}} / D^{\prime} \vDash$ $\langle n, p, q\rangle$. In [25] Quackenbush has shown for Boolean algebras $\mathfrak{B}_{0}, \mathfrak{B}_{1}$ that $\mathfrak{B}_{0}\left[\mathfrak{B}_{1}\right]^{*} \cong \mathfrak{B}_{0} * \mathfrak{B}_{1}$ where $\mathfrak{B}_{0} * \mathfrak{B}_{1}$ is the free product of these Boolean algebras. In view of Burris [3] (Theorem 2.7 and Corollary 2.8) this shows that $\langle n, p, q\rangle \times\langle r, s, t\rangle=$ the elementary characteristic of $\mathfrak{B}_{0} * \mathfrak{B}_{1}$ where $\mathfrak{B}_{0} \vDash\langle r, s, t\rangle$ and $\mathfrak{B}_{1} \vDash\langle n, p, q\rangle$. This yields the result [31] ([9]) that $\times$ is commutative and $\left(\mathfrak{A}\left[\mathfrak{B}_{0}\right]^{*}\right)\left[\mathfrak{B}_{1}\right]^{*} \cong \mathfrak{U}\left[\mathfrak{B}_{1} * \mathfrak{B}_{2}\right]^{*}$ [3] (Corollary 2.8). A complete multiplication table for $\times$ as a commutative semigroup is given in [23] ([24]) and will be extremely useful for some of our proofs. In view of the above, we will sometimes use $\mathfrak{U}[\langle n, p, q\rangle]^{*}$ to denote $\mathfrak{A}[\mathfrak{B}]^{*}$ where $\mathfrak{B} \vDash\langle n, p, q\rangle$.

The next result is rather unfortunate since it severely restricts the theories of $\mathfrak{A}[\mathfrak{B}]$ for infinite $\mathfrak{A}$. 
LEMMA 1.2. If $\mathfrak{B}$ is a complete Boolean algebra, then its elementary characteristic is $\langle 0, p, q\rangle$ for some $p$ and $q$.

Proof. A Boolean algebra $\mathfrak{B}$ has elementary characteristic of the form $\langle 0, p, q\rangle$ iff for each $b$ in $\mathfrak{B}$ the supremum of the atoms below $b$ exist. The latter condition holds in any complete Boolean algebra.

The next results give some insight on Problem 7 of [3] (p. 358).

LEMMA 1.3. There is a sentence preserved by all Boolean powers which is not equivalent to a disjunction of Horn sentences.

Proof. We observe first that for any elementary characteristic $\langle 0, p, q\rangle$ there are complete Boolean algebras having elementary characteristic $\langle 0, p, q\rangle$. In view of [7] or [27] since the property of being complete is preserved by arbitrary direct products, it is sufficient to check that there is a complete Boolean algebra of elementary characteristic $\langle 0,0,1\rangle$. But by [13] (p. 25) the regular open algebra of the Cantor space is complete and atomless since the clopen sets form a basis of open sets. ${ }^{1}$

Let $L$ be the language of Boolean algebras and let $\varphi$ be the sentence of $L$ which asserts that any model $\mathfrak{A}$ of $\varphi$ is a nontrivial Boolean algebra with an atomless element and has elementary characteristic of the form $\langle 0, p, q\rangle$ [7], [6], or [17]. Let $\mathfrak{A}$ be a model of $\varphi$ and consider $\mathfrak{A}[\mathfrak{B}]$. Since $\mathfrak{A}$ is infinite by Definition $1.1, \mathfrak{B}$ must be complete and, hence, $\mathfrak{B}$ has elementary characteristic $\langle 0, p, q\rangle$. By $[2], \mathfrak{A}[\mathfrak{B}] \equiv \mathfrak{A}[\mathfrak{B}]^{*}$ which is isomorphic to $\mathfrak{B}[\mathfrak{U}]^{*}$ by [3] (Corollary 2.8). Using results about combining elementary characteristics at level 0 [7], [29], or [23], it is easy to verify that $\mathfrak{A}[\mathfrak{B}] \vDash \varphi$.

In order to see that $\varphi$ is not equivalent to a disjunction of Horn sentences, it is sufficient to show that it is not preserved by a bounded Boolean power [3] (Theorem 4.3 (vii)). Let $\mathfrak{B}_{0}$ have elementary characteristic $\langle 0, p, 1\rangle$ with $p \neq 0$ and let $\mathfrak{B}_{1}$ have elementary characteristic $\langle 1,1,0\rangle$. Clearly, $\mathfrak{B}_{0} \vDash \varphi$ while $\mathfrak{B}_{0}\left[\mathfrak{B}_{1}\right]^{*} \cong \mathfrak{B}_{1}\left[\mathfrak{B}_{0}\right]^{*}[3]$ (Corollary 2.8) which has elementary characteristic $\langle 1, p, 0\rangle$ by [7], [29], or [23]. Hence, $\mathfrak{B}_{0}\left[\mathfrak{B}_{1}\right]^{*}$ $\vDash \neg \varphi$.

REMARK 1.4. We point out that in [1] a more general notion of $\mathfrak{A}[\mathfrak{B}]$ has been defined since the condition that $\mathfrak{B}$ is complete may be considered too restrictive. It is obvious that for $|\mathfrak{A}|$ denumerable it is sufficient

\footnotetext{
${ }^{1}$ The author thanks E. W. Madison for this observation and for other conversations concerning this paper.
} 
to assume that $\mathscr{B}$ is $\sigma$-complete [13], and, in general, for $\|\mathfrak{a}\|=\kappa$, it is sufficient to assume $\mathfrak{B}$ is $\kappa^{+}$-complete (which means that the supremum of $\kappa$ or fewer elements of $\mathfrak{B}$ exists in $\mathfrak{B}$ ). Most results of [3] hold for this alternative definition of Boolean power. Since we do not know whether or not there are $\sigma$-complete Boolean algebras of elementary characteristic other than $\langle 0, p, q\rangle$, we do not consider in this paper this alternative.

Question. What are the elementary characteristics of Boolean algebras which are $\sigma$-algebras? ${ }^{2}$

The next definition will be fundamental for many of our results; the notion of $B$-separating occurs in [3] (p. 347).

Definition 1.5. $\mathfrak{A}$ is $B$-separating if for all $\mathfrak{B}_{0}, \mathfrak{B}_{1}, \mathfrak{A}\left[\mathfrak{B}_{0}\right]^{*} \cong \mathfrak{A}\left[\mathfrak{B}_{1}\right]^{*}$ implies $\mathfrak{B}_{0} \cong \mathfrak{B}_{1}$. $\mathfrak{A}$ is $T$-separating if all $\mathfrak{B}_{0}, \mathfrak{B}_{1}, \mathfrak{U}\left[\mathfrak{B}_{0}\right]^{*} \equiv \mathfrak{A}\left[\mathfrak{B}_{1}\right]^{*}$ implies $\mathfrak{B}_{0} \equiv \mathfrak{B}_{1}$.

We introduce for a given first-order language $L$ with relation symbols a corresponding language $L_{f}$ with only function symbols. This will enable us to adopt many of the results in [3] to our setting.

Let $L$ be a first-order language with relation symbols and perhaps some function symbols. $L_{f}$ has two new constant symbols $c_{0}$ and $c_{1}$ and has as function symbols all the function symbols of $L$ together with a new $n$-ary function symbol $\mathfrak{X}_{R}$ for each $n$-ary relation symbol $R$ in $L$. For $\mathfrak{U}$ a structure for $L$ with more than one element we define a corresponding structure $\mathfrak{A}_{f}$ for $L_{f}$ as follows: First $\left|\mathfrak{A}_{f}\right|=|\mathfrak{A}|$ and $c_{0}^{\mathfrak{A}_{f}}$ and $c_{1}^{\mathfrak{A}_{f}}$ are chosen to be distinct elements of $\mathfrak{A}$. For each function symbol $f$ of $L_{f}$ which also occurs in $L$ we define $f^{\mathfrak{A}_{f}}=f^{\mathfrak{A}}$. For each $n$-ary function symbol $\mathfrak{X}_{R}$ of $L_{f}$ introduced to replace the $n$-ary relation symbol $R$ of $L$ we define

$$
\begin{array}{ll}
\mathfrak{X}_{R}^{\mathfrak{A}_{f}}\left(a_{1}, \ldots, a_{n}\right)=c_{1}^{\mathfrak{A}_{f}}, & \text { if }\left(a_{1}, \ldots, a_{n}\right) \in R^{\mathfrak{A}} \\
\mathfrak{X}_{R}^{\mathfrak{U}_{f}}\left(a_{1}, \ldots, a_{n}\right)=c_{0}^{\mathfrak{A}_{f}}, & \text { if }\left(a_{1}, \ldots, a_{n}\right) \notin R^{\mathfrak{A}} .
\end{array}
$$

Clearly $\mathfrak{A}_{f}$ satisfies $c_{1} \neq c_{0}$ as well as $\forall x_{1} \cdots \forall x_{n}\left(\mathfrak{X}_{R}\left(x_{1}, \ldots, x_{n}\right)=c_{1} \vee\right.$ $\left.\mathfrak{X}_{R}\left(x_{1}, \ldots, x_{n}\right)=c_{0}\right)$ for each $R$ in $L$. Conversely, any structure $\mathbb{E}$ for $L_{f}$ satisfying these sentences determines a unique $\mathfrak{A}$ for $L$ such that $\mathfrak{U}_{f}=\mathfrak{S}$.

\footnotetext{
${ }^{2}$ Professor Fred Galvin has provided an answer to this question in [11]. He has an extremely elegant argument which shows that for any infinite cardinal $\kappa$ and any Boolean algebra $\mathfrak{B}$ there is a $\kappa$-complete field of sets which is elementarily equivalent to $\mathfrak{B}$. Galvin also obvserved that under the hypothesis that $\kappa$ is measurable Ershov's construction in [7] also yields $\kappa$-complete Boolean algebras of any given elementary characteristic. In view of Galvin's result one can conclude that a sentence is preserved by all these "Boolean powers" of [1] iff it is equivalent to a disjunction of Horn sentences.
} 
LEMMA 1.6. Let $\mathfrak{A}$ and $\mathfrak{A}_{f}$ be as above and $R$ an $n$-ary relation symbol of $L$.

(a) $\mathfrak{A}[\mathfrak{B}]^{*} \vDash R\left[\xi_{1}, \ldots, \xi_{n}\right]$ iff $\mathfrak{A}_{f}[\mathfrak{B}]^{*} \vDash \mathfrak{X}_{R}\left[\xi_{1}, \ldots, \xi_{n}\right]=c_{1}$.

(b) For any proper filter $D$ on $I$,

$$
\begin{aligned}
& \prod_{i \in I} \mathfrak{U}\left[\mathfrak{B}_{i}\right]^{*} / D \vDash R\left[\xi_{1} / D, \ldots, \xi_{n} / D\right] \\
& \quad \text { iff } \prod_{i \in I} \mathfrak{A}_{f}\left[\mathfrak{B}_{i}\right]^{*} / D \vDash \mathfrak{X}_{R}\left[\xi_{1} / D, \ldots, \xi_{n} / D\right]=c_{1} .
\end{aligned}
$$

Proof. The proof of (a) is straightforward and (b) follows easily from (a).

The following results of Burris [3] now hold in our setting.

THEOREM 1.7. (a) If $\mathfrak{A}$ is finite and $D$ is any proper filter on I, then $\Pi_{i \in I} \mathfrak{U}\left[\mathfrak{B}_{i}\right] / D \cong \mathfrak{A}\left[\Pi_{l \in I} \mathfrak{B}_{i} / D\right]$.

(b) If $\mathfrak{U}$ is finite and B-separating, then $\mathfrak{A}$ is T-separating.

Proof. Part (a) with $\mathfrak{A}$ replaced by $\mathfrak{U}_{f}$ follows by [3] (Proposition 2.6) and from this and Lemma 1.6(b) one deduces part (a) as stated.

Part (b) follows exactly as in [3] (Theorem 4.3(x)) using part (a).

The next result is useful for later results and an example is given to show that its converse it not true.

Corollary 1.8. $\sigma: \mathfrak{A}_{f}\left[\mathfrak{B}_{0}\right]^{*} \cong \mathfrak{A}_{f}\left[\mathfrak{B}_{1}\right]^{*}$ implies $\sigma: \mathfrak{A}\left[\mathfrak{B}_{0}\right]^{*} \cong \mathfrak{A}\left[\mathfrak{B}_{1}\right]^{*}$. Hence, if $\mathfrak{U}$ is B-separating, then $\mathfrak{U}_{f}$ is B-separating.

Proof. The first part is immediate by Lemma 1.6(a) and the second part is immediate from the first.

EXAMPLE 1.9. Let $\mathfrak{U}=\langle\{a, b\},\{(a, a)\}\rangle$ be a structure for $L$ consisting of a single binary relation $R$. Let $c_{1}^{\mathfrak{A}_{f}}=b$ and $c_{0}^{\mathfrak{A}_{f}}=a . \mathfrak{U}_{f}$ is $B$-separating by [3] (Theorem 3.4) since it is term equivalent to the 2-element Boolean algebra by [18] (p. 27). However, for any infinite Boolean algebra $\mathfrak{B}$, the interpretation of $R$ in $\mathfrak{A}[\mathfrak{B}]^{*}$ is $\left\{\left\langle\xi_{0}, \xi_{0}\right\rangle\right\}$ where $\xi_{0}(a)=1$ and, hence, the theory of $\mathfrak{U}[\mathfrak{B}]^{*}$ is categorical in all infinite powers. Consequently, by letting $\mathfrak{B}_{0}$ and $\mathfrak{B}_{1}$ be two Boolean algebras of the same cardinality and different complete theories, one obtains $\mathfrak{A}\left[\mathfrak{B}_{0}\right]^{*} \cong \mathfrak{A}\left[\mathfrak{B}_{1}\right]^{*}$ while $\mathfrak{A}_{f}\left[\mathfrak{B}_{0}\right]^{*} \not \mathfrak{A}_{f}\left[\mathfrak{B}_{1}\right]^{*}$ and $\mathfrak{B}_{0} \not \mathfrak{B}_{1}$. 
2. Recursive models. In this section we construct for any recursive structure $\mathfrak{A}$ a recursive model of $\operatorname{Th}\left(\mathfrak{H}^{I} / D\right)$. Using [3] this can be carried out easily as follows. By [7] any elementary characteristic $\langle n, p, q\rangle$ determines a complete theory of a Boolean algebra and this complete theory is decidable. Consequently, if $2^{I} / D \vDash\langle n, p, q\rangle$, then there is a recursive model $\mathfrak{B}(\langle n, p, q\rangle)$ of that elementary characteristic. Given that $\mathfrak{A}$ is recursive, it is easy to see that $\mathfrak{A}[\mathfrak{B}(\langle n, p, q\rangle)]^{*}$ is recursive by definition in [26] and $\mathfrak{U}[\mathfrak{B}(\langle n, p, q\rangle)]^{*} \equiv \mathfrak{A}^{I} / D$ by [3] (Theorem 4.3(i) and (v)).

One can also be very explicit as to the construction of a recursive $\mathfrak{B}(\langle n, p, q\rangle)$. In fact in [16] recursive prime models of each elementary characteristic are constructed explicitly in terms of an ordered basis. The prime models are the smallest countable models of these theories in contrast to the countable saturated models in [23] which are the largest countable models of these theories. Moreover, the models of [16] have recursive satisfaction relation and the property that $\mathfrak{A}$ has a recursive satisfaction relation will be preserved by these particular bounded Boolean powers by the Feferman-Vaught-Weinstein Theorem. Next we give an example of the models of $\operatorname{Th}\left(\mathfrak{H}^{\omega}\right)$ obtained using [16] from the point of view of [22].

EXAMPLE 2.1. Let $\mathfrak{B}(\langle 0, \omega, 0\rangle)$ denote the substructure of $2^{\omega}$ with domain consisting of all functions in $2^{\omega}$ which are eventually constant. It follows by [16] that $\mathfrak{B}(\langle 0, \omega, 0\rangle)$ is the prime model of $\operatorname{Th}\left(2^{\omega}\right)$ and by [7] (Theorem 3) that $\mathfrak{B}(\langle 0, \omega, 0\rangle) \prec 2^{\omega}$. Let $\mathfrak{E}$ be the substructure of $\mathfrak{A}^{\omega}$ with domain consisting of all functions in $\mathfrak{A}^{\omega}$ which are eventually constant. It follows that $\mathfrak{E} \cong \mathfrak{A}[\mathfrak{B}(\langle 0, \omega, 0\rangle)]^{*}$ and that $\mathbb{E} \prec \mathfrak{U}^{\omega}[3]$ (Theorem 4.3(v)).

3. Applications to logic of reduced power structures. In [20] for each elementary characteristic $\langle n, p, q\rangle$ of a complete theory of a Boolean algebra and any first-order language $L$, we defined semantically $V(\langle n, p, q\rangle)$ the set of valid sentences of $L$ of reduced power structures of type $\langle n, p, q\rangle$. Also we gave a syntactic characterization of $V(\langle n, p, q\rangle)$ [20] (Theorem 1.4) and proved the following [20] (Theorem 1.6):

THEOREM 3.1. $\mathfrak{A} \vDash V(\langle n, p, q\rangle)$ iff for some $\mathfrak{E}, \mathfrak{A} \equiv \mathfrak{C}^{I} / D$ where $2^{I} / D$ $\vDash\langle n, p, q\rangle$.

In [20] (\$3) we demonstrated numerous results about the logics $V(\langle n, p, q\rangle)$ and the more interesting of these results required that $L$ be sufficiently large, i.e., contain the language of Boolean algebras. In this 
section we intend to show that all of the results of [20] (\$3) hold under the hypothesis that $L$ contains a binary relation. It is sufficient to consider $L$ with exactly one binary relation denoted by $R$.

Let $\mathfrak{A}_{2}$ be a structure for $L$ where $\left|\mathfrak{A}_{2}\right|=\{a, b\}, a \neq b$ and $R^{\mathfrak{A}_{2}}=$ $\{(a, a),(b, b),(a, b)\}$, i.e., $\mathfrak{A}_{2}$ is a linear order of order type 2 . We are interested in the theories obtained by taking reduced powers of $\mathfrak{A}_{2}$ and it is sufficient to consider $\mathfrak{H}_{2}[\mathfrak{B}]^{*}$. We observe first that $\mathfrak{U}_{2}[\mathfrak{B}]^{*}$ is a partially ordered set which is a distributive complemented lattice with a first and a last element.

\section{LEMMA 3.2. $\mathfrak{A}_{2}$ is B-separating.}

Proof. Let $\mathfrak{B}$ be a Boolean algebra and consider $\mathfrak{A}_{2}[\mathfrak{B}]^{*}$. It is not difficult to see that $\mathfrak{U}_{2}[\mathfrak{B}]^{*}$ is isomorphic to $\mathfrak{B}$ as a partially ordered set, i.e. isomorphic to $\left\langle B, \leq^{\mathfrak{B}}\right\rangle$ where for $x, y$ in $B, x \leq^{B} y$ iff $x \cap y=x$ in $\mathfrak{B}$. Suppose $\mathfrak{A}_{2}\left[\mathfrak{B}_{0}\right]^{*} \cong \mathfrak{A}_{2}\left[\mathfrak{B}_{1}\right]^{*}$, then $\left\langle B_{0}, \leq^{\mathfrak{B}_{0}}\right\rangle \cong\left\langle\boldsymbol{B}_{1}, \leq^{\mathfrak{B}_{1}}\right\rangle$ and, hence, $\mathfrak{B}_{0} \cong \mathfrak{B}_{1}$ since the lattice structure of $\mathfrak{B}_{0}$ determines $\mathfrak{B}_{0}$ up to isomorphism.

COROllary 3.3. $\mathfrak{A}_{2}$ is T-separating.

Proof. This is immediate by Theorem 1.7(b).

Let $\left(\mathfrak{A}_{2}\right)_{f}$ be defined as in $\S 1$.

Corollary 3.4. $\left(\mathfrak{A}_{2}\right)_{f}$ is B-separating.

Proof. This is immediate by Corollary 1.8 .

The next result demonstrates that the theory of distributive complemented lattices behaves like a universal Horn theory.

LEMMA 3.5. If $\mathfrak{A}$ is a structure for $L$ and $\mathfrak{A}^{I} / D$ is a distributive complemented lattice with first and last elements, then $\mathfrak{A}$ is a distributive complemented lattice with first and last elements.

Proof. First note that $\mathfrak{A}$ is embeddable in $\mathfrak{A}[\mathfrak{B}]^{*}$ via $a \rightarrow f_{a}$ where $f_{a}(a)=1$. Since $\mathfrak{A}[\mathfrak{B}]^{*}$ is a partially ordered set, so is $\mathfrak{A}$ since the property of being a partially ordered set via $R$ can be expressed by a universal (Horn) sentence of $L$. One can argue directly that in $\mathfrak{A}[\mathfrak{B}]^{*}$, $f_{a} \vee f_{b}=f_{c}$ for some $c$ in $|\mathfrak{U}|$ and $f_{a} \wedge f_{b}=f_{d}$ for some $d$ in $|\mathfrak{A}|$. Moreover, 
the first and last elements of $\mathfrak{A}[\mathfrak{B}]^{*}$ are also of the form $f_{a}$ and $f_{b}$ for some $a$ and $b$ in $|\mathfrak{A}|$. The distributive complemented property in $\mathfrak{A}$ follows readily.

The next result allows us to generaize the results [20] (§3) using exactly the same reasoning as in [20]. For our notation and background see our comments prior to Lemma 1.2.

LEMMA 3.6. For the language $L=\langle R\rangle, V(\langle r, s, t\rangle) \subseteq V(\langle n, p, q\rangle)$ iff there exists $\left\langle n^{\prime}, p^{\prime}, q^{\prime}\right\rangle$ such that $\langle n, p, q\rangle=\left\langle n^{\prime}, p^{\prime}, q^{\prime}\right\rangle \times\langle r, s, t\rangle$.

Proof. Suppose $\langle n, p, q\rangle=\left\langle n^{\prime}, p^{\prime}, q^{\prime}\right\rangle \times\langle r, s, t\rangle$. Let $\mathfrak{M} \vDash$ $V(\langle n, p, q\rangle)$. By Theorem 3.1, $\mathfrak{M} \equiv \mathfrak{C}^{I} / D$ such that $2^{I} / D \vDash\langle n, p, q\rangle$. But $\left(\Im^{J} / E\right)^{I^{\prime}} / D^{\prime} \equiv \mathfrak{\Im}^{I} / D$ where $2^{I^{\prime}} / D^{\prime} \vDash\left\langle n^{\prime}, p^{\prime}, q^{\prime}\right\rangle$ and $2^{J} / E \vDash\langle r, s, t\rangle$. Hence $\mathfrak{M} \vDash V(\langle r, s, t\rangle)$ by commutativity and Theorem 3.1.

Conversely, suppose $V(\langle r, s, t\rangle) \subseteq V(\langle n, p, q\rangle)$. Let $\mathfrak{B}$ be a Boolean algebra of elementary characteristic $\langle n, p, q\rangle$ and consider $\mathfrak{A}_{2}[\mathfrak{B}]^{*}$, i.e., $\mathfrak{A}_{2}[\mathfrak{B}]^{*} \equiv \mathfrak{U}_{2}^{I} / D$, where $2^{I} / D \vDash\langle n, p, q\rangle$. Thus by Theorem $3.1, \mathfrak{A}_{2}[\mathfrak{B}]^{*}$ $\vDash V(\langle n, p, q\rangle)$ and, hence $\mathfrak{U}_{2}[\mathfrak{B}]^{*} \equiv \mathfrak{C}^{J} / E$ where $2^{J} / E \vDash\langle r, s, t\rangle$. By Lemma 3.5, $\mathbb{C}$ is a distributive complemented lattice with first and last elements. Hence, $\mathfrak{C}=\left\langle B_{0}, \leq^{\mathfrak{B}_{0}}\right\rangle$ where $\mathfrak{B}_{0}$ has the elementary characteristic $\left\langle n^{\prime}, p^{\prime}, q^{\prime}\right\rangle$. Thus, $\mathfrak{U}_{2}[\mathfrak{B}]^{*} \equiv\left(\mathfrak{A}_{2}\left[\mathfrak{B}_{0}\right]^{*}\right)\left[2^{J} / E\right]^{*}$ so $\mathfrak{A}_{2}[\mathfrak{B}]^{*} \equiv$ $\mathfrak{A}_{2}\left[\mathfrak{B}_{0} * 2^{J} / E\right]^{*}$. By Corollary 3.3, $\mathfrak{B} \equiv \mathfrak{B}_{0} *\left[2^{J} / E\right]$ and, hence, $\langle n, p, q\rangle=\left\langle n^{\prime}, p^{\prime}, q^{\prime}\right\rangle \times\langle r, s t\rangle$ by commutativity.

THEOREM 3.7. For any language $L$ containing a binary relation symbol ( $n$-ary for $n \geq 2), V(\langle n, p, q\rangle)$ are all different and are partially ordered under $\subseteq$ exactly as in [20] (also see [23]).

A similar result holds for all of the assertions in [20] (§3).

\section{Axiomatizations over $\mathcal{H}(\mathfrak{U})$.}

DeFINITION 4.1. $\mathcal{H}(\mathfrak{A})=\{\varphi \mid \varphi$ is a Horn sentence and $\mathfrak{A} \vDash \varphi\}$.

In this section we show that if $\mathfrak{U}$ is $T$-separating, then the complete extensions of $\mathscr{H}(\mathfrak{H})$ behave exactly as the complete theories of Boolean algebras with respect to finitely axiomatizability over $\mathcal{H}(\mathfrak{H})$. Next we show that for any $\mathfrak{A}, \operatorname{Th}(\mathfrak{H})$ is finitely axiomatizable over $\mathcal{H}(\mathfrak{H})$. This improves a result of Galvin [10] (Theorem 9.1(a)). 
The following combinatorial result is in [6] (Exercise 6.2.3).

LEMmA 4.2. $\prod_{i \in I}\left(\mathfrak{U}^{I_{i}} / D_{i}\right) / D \cong \Pi_{i \in K} \mathfrak{U} / E$, where $2^{K} / E \cong$ $\prod_{i \in I}\left(2^{I_{i}} / D_{i}\right) / D$.

The next result shows that $\mathcal{H}(\mathfrak{H})$ has only countably many complete extensions, each of which is the theory of a reduced power of $\mathfrak{A}$.

LEMMA 4.3. $\mathfrak{M} \vDash \mathcal{H}(\mathfrak{A})$ iff for some $I$ and $D, \mathfrak{M} \equiv \mathfrak{A}^{I} / D$.

Proof. By Lemma 4.2, it follows that $\{\mathfrak{M} \mid$ for some $I$ and $D$, $\left.\mathfrak{M} \equiv \mathfrak{A}^{I} / D\right\}$ is an elementary class since it is closed under ultra-products. Thus, any $\mathfrak{M}$ which is a model of $\operatorname{Th}\left\{\mathfrak{M} \mid\right.$ for some $I$ and $\left.D, \mathfrak{M} \equiv \mathfrak{U}^{I} / D\right\}$ is elementarily equivalent to $\mathfrak{A}^{I} / D$ for some $I$ and $D$. Hence, $\mathfrak{M}$ is a model of $\mathcal{H}(\mathfrak{U})$ by [6] (Proposition 6.2.2). Suppose $\mathfrak{M}$ is a model of $\mathcal{H}(\mathfrak{A})$, then any Horn sentence true in $\mathfrak{A}$ holds in $\mathfrak{M}$ and, hence, some elementary extension of $\mathfrak{M}$ is isomorphic to a proper reduced power of $\mathfrak{A}$ [6] (Lemma 6.2.4). Thus, $\mathfrak{M} \equiv \mathfrak{A}^{I} / D$ for some $I$ and $D$.

DEFINITION 4.4. Let $T$ be a theory and $T^{\prime}$ be a complete extension of $T$. $T^{\prime}$ is finitely axiomatizable over $T$ if there is a sentence $\varphi$ such that $T^{\prime}$ is equivalent to $T \cup\{\varphi\}$.

The following result is analogous to [6] (Theorem 4.1.12).

LEMMA 4.5. $T^{\prime}$ is finitely axiomatizable over $T$ iff $\{\mathfrak{M} \mid \mathfrak{M} \vDash T$ and not $\left.\mathfrak{M} \vDash T^{\prime}\right\}$ is closed under ultra-products.

The next result is implicit in Ershov [7].

LEMMA 4.6. The finitely axiomatizable complete theories of Boolean algebras are those of elementary characteristic $\langle n, p, q\rangle$ where $p \neq \omega$ and $n \neq \omega$.

We can deduce from [3] (Theorem 4.3) that $\mathfrak{A}$ is $T$-separating iff $\mathfrak{A}^{I} / D \equiv \mathfrak{U}^{J} / E$ implies $2^{I} / D \equiv 2^{J} / E$. Using the above machinery we can now show the next result.

THEOREM 4.7. If $\mathfrak{A}$ is $T$-separating, then $\mathfrak{A}^{I} / D$ is finitely axiomatizable over $\mathcal{H}(\mathfrak{U})$ iff $2^{I} / D$ is a finitely axiomatizable Boolean algebra. 
Proof. Consider an ultra-product of models $\mathfrak{U}^{I_{i}} / D_{i}$ of $\mathcal{H}(\mathfrak{U})$ which are not elementarily equivalent to $\mathfrak{A}^{I} / D$. By Lemma 4.2, $\Pi_{i \in I^{\prime}}\left(\mathfrak{A}^{I_{i}} / D_{i}\right) / D^{\prime} \cong \Pi_{l \in J} \mathfrak{A} / E$ where $2^{J} / E \cong \Pi_{i \in I^{\prime}}\left(2^{I_{i}} / D_{i}\right) / D^{\prime}$. Suppose $2^{I} / D$ is finitely axiomatizable, then since $\mathfrak{A}^{I_{i}} / D_{i} \neq \mathfrak{A}^{I} / D$, we know $2^{I_{i}} / D_{l} Z 2^{I} / D$ since $\mathfrak{A}$ is $T$-separating. Since $2^{I} / D$ is finitely axiomatizable, it follows that $\prod_{i \in I^{\prime}}\left(2^{I_{i}} / D_{i}\right) / D^{\prime} \neq 2^{I} / D$. Thus, $2^{J} / E Z 2^{I} / D$ and, hence, $\mathfrak{A}^{J} / E \neq \mathfrak{A}^{I} / D$ since $\mathfrak{A}$ is $T$-separating. Thus, by Lemma $4.5 \mathfrak{A}^{I} / D$ is finitely axiomatizable over $\mathscr{H}(\mathfrak{A})$. Suppose $2^{I} / D$ is not finitely axiomatizable, then for some $2^{I_{i}} / D_{i} \neq 2^{I} / D$ and ultra-filter $D^{\prime}$ over $I^{\prime}$ we have $\left(\prod_{l \in I^{\prime}} 2^{I_{i}} / D_{i}\right) / D^{\prime} \equiv 2^{I} / D$. Consequently, $\prod_{i \in I^{\prime}}\left(\mathfrak{U}^{I} / D_{i}\right) / D^{\prime} \cong \mathfrak{U}^{J} / E \equiv$ $\mathfrak{A}^{I} / D$. Thus, there is an ultra-product of models of $\mathcal{H}(\mathfrak{U})$ each of which is not elementarily equivalent to $\mathfrak{H}^{I} / D$ such that the ultra-product is elementarily equivalent to $\mathfrak{H}^{I} / D$. Thus, by Lemma $4.5 \mathfrak{H}^{I} / D$ is not finitely axiomatizable over $\mathcal{H}(\mathfrak{A})$.

EXAMPLE 4.8. Let $\omega=\langle\omega,+, \cdot, 0,1\rangle . \omega$ is $T$-separating since in $\omega^{I} / D$ the set $\left\{x \mid \omega^{I} / D \vDash x \cdot x=x\right\}$ forms a partially ordered set under $x \leq y$ defined by $x \cdot y=x$ which is isomorphic to the partial order of $2^{I} / D$. Thus, $\omega$ is finitely axiomatizable over $\mathcal{H}(\omega)$ and clearly $\operatorname{Th}(\omega)$ is axiomatized by $\mathcal{H}(\omega) \cup\{\forall x \forall y(x \cdot y=0 \Rightarrow x=0 \vee y=0)\}$.

We remark that $\langle\omega, \cdot\rangle$ is also $B$-separating and $T$-separating by the above observations. Also, $\langle\omega,+\rangle$ is $B$-separating and $T$-separating, see [28].

It has been shown in [12] that there are $B$-separating structures $\mathfrak{A}$ which are not $T$-separating. The next result is an easy consequence of known results.

Lemma 4.9. If $\mathfrak{A}$ is $T$-separating, then $\mathfrak{A}^{k}$ is $T$-separating for $1<k<\omega$. If $\mathfrak{A}$ is B-separating, then $\mathfrak{U}^{k}$ is not B-separating for $1<k<\omega$.

Proof. $\mathfrak{B}^{k}$ has elementary characteristic $\langle n, k \cdot p, q\rangle$ if $\mathfrak{B} \vDash\langle n, p, q\rangle$ where $k \cdot \omega=\omega$ by [7] or [29]. Since $\mathfrak{A}$ is $T$-separating, so must also be $\mathfrak{A}^{k}$, since by [3] (Proposition 2.1 (iv)), $\mathfrak{A}^{k}[\mathfrak{B}]^{*} \cong \mathfrak{U}\left[\mathfrak{B}^{k}\right]^{*}$. Also by [14] (Theorem 2) there are denumerable Boolean algebras $\mathbb{E}$ and $\mathfrak{D}$ such that $\mathfrak{C}^{k} \cong \mathfrak{D}^{k}$ but $\mathfrak{E} \not \mathfrak{D}$. Hence $\mathfrak{A}^{k}[\mathfrak{C}]^{*} \cong \mathfrak{U}^{k}[\mathfrak{D}]^{*}$ but $\mathfrak{C} \neq \mathfrak{D}$.

Now we give two preliminary results about ways in which $\mathfrak{A}$ can fail to be $T$-separating and we will proceed to the last main result of this section.

Lemma 4.10. $\mathfrak{A} \equiv \mathfrak{A} \times \mathfrak{A}$ iff $\mathfrak{A} \equiv \prod_{i \in \omega} \mathfrak{A}$. 
Proof. Suppose $\mathfrak{A} \equiv \mathfrak{U} \times \mathfrak{A}$. Then by induction $\mathfrak{A}^{n} \equiv \mathfrak{A}$ for $n \geq 1$. Hence by [8], $\mathfrak{A} \equiv \prod_{i \in \omega} \mathfrak{A}$. Suppose $\mathfrak{A} \equiv \prod_{i \in \omega} \mathfrak{A}$. Then since $\Pi_{i \in \omega} \mathfrak{A} \times$ $\prod_{i \in \omega} \mathfrak{A} \cong \prod_{i \in \omega} \mathfrak{A}$, it follows that $\mathfrak{A} \times \mathfrak{A} \equiv \mathfrak{U}$.

LEMMA 4.11. If $\mathfrak{U}^{p}=\mathfrak{U}^{q}$ for some $1 \leq p<q<\omega$, then there is a smallest $k$ such that $\mathfrak{A}^{k} \equiv \prod_{i \in \omega} \mathfrak{A}$.

Proof. $\mathfrak{A}^{p} \equiv \mathfrak{U}^{q} \cong \mathfrak{U}^{p} \times \mathfrak{U}^{q-p} \equiv \mathfrak{U}^{q} \times \mathfrak{U}^{q-p} \equiv \mathfrak{U}^{p} \times \mathfrak{U}^{2(q-p)}$. Thus, by induction $\mathfrak{U}^{p} \equiv \mathfrak{A}^{p} \times \mathfrak{A}^{n(q-p)}$ for $n \geq 1$. Thus, $\mathfrak{A}^{p} \equiv \Pi_{i \in \omega} \mathfrak{A}$ by [8]. Hence, $\mathfrak{U}^{p} \equiv \mathfrak{A}^{n}$, for $n \geq p$. Thus there is a smallest such $p$.

Recall the comments prior to Lemma 1.2 for the definition of $\mathfrak{U}[\langle n, p, q\rangle]^{*}$.

THEOREM 4.12. $\operatorname{Th}(\mathfrak{A})$ is finitely axiomatizable over $\mathcal{H}(\mathfrak{A})$.

Proof. Case 1. $\mathfrak{A}$ is $T$-separating. The result is immediate by Theorem 4.7.

Case 2. $\mathfrak{U} \neq \mathfrak{A} \times \mathfrak{A}$. The strategy will be to write down a list of axioms for $\mathrm{Th}(\mathfrak{A})$. By hypothesis, the proof of Lemma 4.11, and Lemma 4.10, $\mathfrak{A} Z \mathfrak{U}^{p}$ for $1<p \leq \omega$. Let $\sigma_{\langle 0, \omega, 0\rangle}$ denote a Horn sentence true in $\mathfrak{A}[\langle 0, \omega, 0\rangle]^{*}$ and false in $\mathfrak{A}$ since $\mathscr{H}(\mathfrak{A}) \subset \mathcal{H}\left(\mathfrak{U}^{\omega}\right)[10]($ Lemma 7.5$)$ and [6] (Proposition 6.2.2). By [8] $\sigma_{\langle 0, \omega, 0\rangle}$ is true in all but infinitely many $\mathfrak{A}^{P}$, $1<p \leq \omega$. For each $p$ such that $\mathfrak{A}^{p} \vDash \neg \sigma_{\langle 0, \omega, 0\rangle}$ select a Horn sentence $\sigma_{\langle 0, p, 0\rangle}$ true in $\mathfrak{U}^{p}$ and false in $\mathfrak{A}$ by the same argument as above. Since $\langle\omega, 0,0\rangle \times\langle 0, \omega, 0\rangle=\langle\omega, 0,0\rangle$, it follows by [6] (Proposition 6.2.2) that $\mathfrak{U}[\langle\omega, 0,0\rangle]^{*} \vDash \sigma_{\langle 0, \omega, 0\rangle}$. By the Feferman-Vaught-Weinstein Theorem, let $\left(\sigma ; \psi_{1}, \ldots, \psi_{n}\right)$ be the determining sequence for $\sigma_{\langle 0, \omega 0\rangle}$ and, hence, $\langle\omega, 0,0\rangle \vDash \sigma\left[c_{1}, \ldots, c_{\mathrm{m}}\right]$ where $c_{i}=\overline{0}$ if $\mathfrak{A} \vDash \neg \psi_{i}$, or $c_{i}=\overline{1}$ if $\mathfrak{A} \vDash \psi_{l}$, for $1 \leq i \leq m$. By [29] (Theorem) for all $\langle n, p, q\rangle$ with $n$ sufficiently large $\langle n, p, q\rangle \vDash \sigma\left[c_{1}, \ldots, c_{m}\right]$ and, hence, $\mathfrak{A}[(\langle n, p, q\rangle)]^{*} \vDash \sigma_{\langle 0, \omega, 0\rangle}$. Clearly, $\mathfrak{U}[\langle 0,0,1\rangle]^{*} \vDash \sigma_{\langle 0, \omega, 0\rangle} ;$ moreover, by easy calculations for $r \geq 0$ and $(p=$ $0 \vee p>1), \quad \mathfrak{A}[\langle r, p, t\rangle]^{*} \vDash \sigma_{\langle 0, \omega, 0\rangle} \vee \sigma_{\langle 0, p, 0\rangle}$ since $\mathfrak{U}[\langle r, p, t\rangle]^{*} \equiv$ $\mathfrak{A}^{p}[\langle r, 1, t\rangle]^{*}$ for $\left.p\right\rangle 1$, and for $p=0, \mathfrak{A}^{\omega}[\langle r, 0,1\rangle]^{*} \equiv \mathfrak{U}[\langle r, 0,1\rangle]^{*}$. Thus, the only models of $T=\mathscr{H}(\mathfrak{A}) \cup\{\neg \sigma \mid \sigma$ is a Horn sentence considered above $\}$ are of the form $\mathfrak{A}[\langle r, 1,1\rangle]^{*}$ or $\mathfrak{A}[\langle r, 1,0\rangle]^{*}$. For each such elementary characteristic $\mathfrak{A}[\langle r, 1, t\rangle]^{*} \not \mathfrak{A}$, find a Horn sentence $\sigma$ true in $\mathfrak{A}[\langle r, 1, t\rangle]^{*}$ and false in $\mathfrak{A}$ and add its negation to $T$. In this way we obtain a finite extension $T^{\prime}$ of $T$ and hence of $\mathscr{H}(\mathfrak{A})$ such that $\mathfrak{A} \vDash T^{\prime}$ implies $\mathfrak{M} \equiv \mathfrak{A}$. 
Case 3. $\mathfrak{U} \equiv \mathfrak{U} \times \mathfrak{A}$. Suppose $\mathfrak{A} \equiv \mathfrak{A}[\langle 0,0,1\rangle]^{*}$. Then $\operatorname{Th}(\mathfrak{A})$ is a complete Horn theory by $[\mathbf{1 0}]$ and, hence, $\operatorname{Th}(\mathfrak{A}) \equiv \mathcal{H}(\mathfrak{A})$. Suppose $\mathfrak{U} \not \mathfrak{U}[\langle 0,0,1\rangle]^{*}$. Let $\sigma_{\langle 0,0,1\rangle}$ be a Horn sentence demonstrating this. If $\mathfrak{U} \equiv \mathfrak{A}[\langle\omega, 0,0\rangle]^{*}$ and $\mathfrak{U} Z \mathfrak{U}[\langle 0,0,1\rangle]^{*}$, then we are done since $\operatorname{Th}(\mathfrak{U})$ is equivalent to $\mathscr{H}(\mathfrak{H}) \cup\left\{\neg \sigma_{\langle 0,0,1\rangle}\right\}$ since $\mathfrak{M} \vDash \mathcal{H}(\mathfrak{H}) \cup\left\{\neg \sigma_{\langle 0,0,1\rangle}\right\}$ implies by Lemma 4.3 that $\mathfrak{M} \equiv \mathfrak{U}^{Y} / D$ where $2^{I} / D \forall\langle 0,0,1\rangle$ and, hence, $\mathfrak{M} \equiv$ $\mathfrak{A}[\langle\omega, 0,0\rangle]^{*} \equiv \mathfrak{U}$ by [24] or [23]. If $\mathfrak{U} Z \mathfrak{U}[\langle\omega, 0,0\rangle]^{*}$ and $\mathfrak{A} Z$ $\mathfrak{U}[\langle 0,0,1\rangle]^{*}$, let $\sigma_{\langle\omega, 0,0\rangle}$ be a Horn sentence true in $\mathfrak{A}[\langle\omega, 0,0\rangle]^{*}$ and false in $\mathfrak{A}$. As in Case 2, by the Feferman-Vaught-Weinstein Theorem and [29], $\sigma_{\langle\omega, 0,0\rangle}$ is true in all $\mathfrak{U}[\langle n, p, q\rangle]^{*}$ for $n$ sufficiently large. Consider $\mathscr{H}(\mathfrak{A}) \cup\left\{\neg \sigma_{\langle\omega, 0,0\rangle}\right\}=T$. There is an $n$ such that if $\mathfrak{M} \vDash T$, then $\mathfrak{M} \equiv$ $\mathfrak{U}^{I} / D$ for some $I$ and $D$ such that $2^{I} / D \vDash\langle r, s, t\rangle$ and $r<n$. By Lemma 4.10, $\mathfrak{A}=\mathfrak{A}^{\omega}$ and, hence $\mathfrak{A}[\langle r, s, t\rangle]^{*} \equiv \mathfrak{A}^{\omega}[\langle r, s, t\rangle]^{*}$. Thus, the models of $T$ which are not elementarily equivalent to $\mathfrak{A}$ are elementarily equivalent to $\mathfrak{A}[\langle r, 0,1\rangle]^{*}, \mathfrak{A}[\langle r, \omega, 0\rangle]^{*}$ or $\mathfrak{A}[\langle r, \omega, 1\rangle]^{*}$ with $r<n$. For each one select a Horn sentence true in it and false in $\mathfrak{A}$; add the negation of each Horn sentence so selected to $T$ to obtain $T^{\prime}$. Hence, $\mathfrak{M} \vDash T^{\prime}$ implies $\mathfrak{M} \equiv \mathfrak{A}$.

\section{REFERENCES}

[1] C. J. Ash, Reduced powers and Boolean extensions, J. London Math. Soc. (2), 9 (1975), 429-432.

[2] B. Banaschewski and E. Nelson, Boolean powers as algebras of continuous functions, Dissertationes Mathematicae, 179 (1980), Warsaw.

[3] S. Burris, Boolean powers, Algebra Universalis, 5 (1975), 340-360.

[4] _ Bounded Boolean powers and $\equiv_{n}$, Algebras Universalis, 8 (1978), 137-138.

[5] S. Burris and H. Werner, Sheaf constructions and their elementary properties, TAMS, 248 (1979), 269-309.

[6] C. C. Chang and H. J. Keisler, Model Theory, North-Holland, Amsterdam, 1973.

[7] Yu. L. Ershov, Decidability of the elementary theory of relatively complemented lattices and the theory of filters, Algebra i Logika, 17 (1964), 17-38. (Russian)

[8] S. Feferman and R. L. Vaught, The first order properties of algebraic systems, Fund. Math., 47 (1959), 57-103.

[9] F. Galvin, Reduced Products, Horn sentences, and decision problems, Bull. Amer. Math. Soc., 73 (1967), 59-64.

[10] Horn sentences, Ann. Math. Logic, 1 (1969), 389-442.

[11] Personal communication.

[12] S. Garavaglia and J. M. Plotkin, Separation properties and Boolean powers, Coll. Math., (to appear).

[13] P. R. Halmos, Lectures on Boolean Algebras, Van Nostrand, Princeton, 1967.

[14] W. Hanf, On some fundamental problems concerning isomorphisms of Boolean algebras, Math. Scand., 5 (1957), 205-217.

[15] J. Lawrence, Boolean powers of groups, Proc. Amer. Math. Soc., 82 (1981), 512-515.

[16] J. Mead, Recursive prime models for Boolean algebras, Coll. Math., 41 (1979), 25-33. 
[17] J. Mead and G. C. Nelson, Model companions and k-model completeness for the complete theories of Boolean algebras, J. Symbolic Logic, 45 (1980), 47-55.

[18] E. Mendelson, Introduction to Mathematical Logic, 2nd ed., D. Van Nostrand, New York, 1979.

[19] A. Mostowski, On direct powers of theories, J. Symbolic Logic, 17 (1952), 1-31.

[20] G. C. Nelson, Logic of reduced power structures, J. Symbolic Logic, 48 (1983), 53-59.

[21] G. C. Nelson, Addendum to "Logic of reduced power structures", J. Symbolic Logic, 48 (1983), 1145. ${ }^{3}$

[22] G. C. Nelson, The periodic power of $Q$ and complete Horn theories, Algebra Universalis, 14 (1982), 349-356.

[23] P. Olin, Free products and elementary type of Boolean algebras, Math. Scand., 38 (1976), 5-23.

[24] A. I. Omarov, On subsystems of reduced powers, Algebra i Logika, 12 (1973), 42-46 (English translation).

[25] R. W. Quackenbush, Free products of bounded distributive lattices, Algebra Universalis, 2 (1972), 393-394.

[26] M. O. Rabin, Computable algebra, Trans. Amer. Math. Soc., 95 (1960), 341-360.

[27] R. Sikorski, Boolean Algebras, Academic Press, New York, 1964.

[28] A. Tarski, Remarks on direct products of commutative semigroups, Math. Scand., 5 (1957), 218-223.

[29] J. Waszkiewicz, $\forall_{n}$-theories of Boolean algebras, Coll. Math., 30 (1974), 171-175.

[30] J. Waszkiewicz and B. Wȩglorz, Some models of theories of reduced powers, Bull. Acad. Polon. Sci. Math. Astron. Phys., 16 (1968), 683-685.

[31] B. Wȩglorz, Substructures of reduced powers, Fund. Math., 89 (1975), 191-197.

[32] V. Weispfenning, Model-completeness and elimination of quantifiers for subdirect products of structures, J. Algebra, 36 (1975), 252-277.

Received April 28, 1982, and in revised form November 9, 1982. The author acknowledges the many helpful suggestions that the referee has contributed to this revision of a previously submitted article.

\section{UNIVERSITY OF IOWA}

IOWA CITY, IA 52242

\footnotetext{
${ }^{3}$ In [21] it is pointed out that Galvin in [9] had announced many of the results in $\$ 2$ of [20].
} 


\section{PACIFIC JOURNAL OF MATHEMATICS \\ EDITORS}

DONALD BABBITt (Managing Editor)

University of California

Los Angeles, CA 90024

J. Dugundu

University of Southern California

Los Angeles, CA 90089-1113

R. FINN

Stanford University

Stanford, CA 94305

HERMANN FLASCHKA

University of Arizona

Tucson, AZ 85721
C. C. MOORE

University of California

Berkeley, CA 94720

ARTHur Ogus

University of California

Berkeley, CA 94720

Hugo RossI

University of Utah

Salt Lake City, UT 84112

H. SAMELSON

Stanford University

Stanford, CA 94305

ASSOCIATE EDITORS

R. ARENS

E. F. BECKENBACH

B. H. NeumanN (1906-1982)

F. WOLF

K. YoshidA

\section{SUPPORTING INSTITUTIONS}

UNIVERSITY OF ARIZONA

UNIVERSITY OF BRITISH COLUMBIA

UNIVERSITY OF OREGON

CALIFORNIA INSTITUTE OF TECHNOLOGY

UNIVERSITY OF CALIFORNIA

MONTANA STATE UNIVERSITY

UNIVERSITY OF SOUTHERN CALIFORNIA

UNIVERSITY OF NEVADA, RENO

STANFORD UNIVERSITY

UNIVERSITY OF HAWAII

NEW MEXICO STATE UNIVERSITY

UNIVERSITY OF TOKYO

UNIVERSITY OF UTAH

OREGON STATE UNIVERSITY

WASHINGTON STATE UNIVERSITY

UNIVERSITY OF WASHINGTON 


\section{Pacific Journal of Mathematics}

Vol. 114, No. $1 \quad$ May, 1984

David Marion Arnold and Charles Irvin Vinsonhaler, Typesets and cotypesets of rank-2 torsion free abelian groups $\ldots \ldots \ldots \ldots \ldots \ldots \ldots 1$

Duncan Alan Buell and Richard Howard Hudson, Solutions of certain

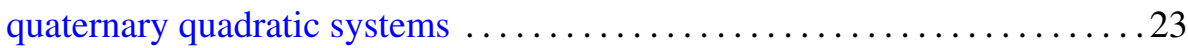

Hans Delfs and Manfred Knebusch, Separation, retractions and homotopy

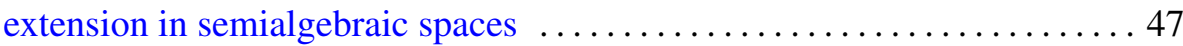

I. Erdélyi and Sheng-Wang Wang, A spectral duality theorem for closed

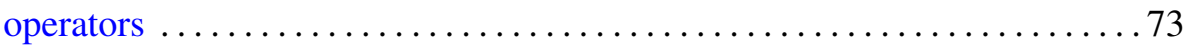

Theodore William Gamelin, Weak compactness of representing measures for $R(K)$

Kenneth R. Goodearl and T. H. Lenagan, Krull dimension of

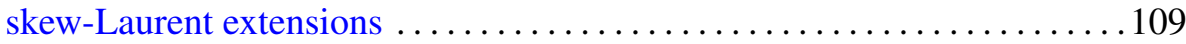

Daniel Joseph Gross, Compact quotients by $\mathbf{C}^{*}$-actions . . . . . . . . . . . 149

Goo Ishikawa, Satoshi Koike and Masahiro Shiota, Critical value sets of

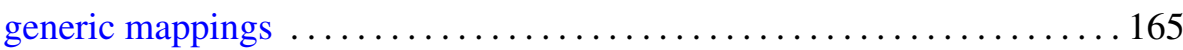

Hong Oh Kim, Derivatives of Blaschke products $\ldots \ldots \ldots \ldots \ldots \ldots \ldots \ldots \ldots$

Erhard Luft and Denis Karmen Sjerve, 3-manifolds with subgroups

$Z \oplus Z \oplus Z$ in their fundamental groups $\ldots \ldots \ldots \ldots \ldots \ldots \ldots \ldots \ldots \ldots \ldots \ldots$

George Clifford Nelson, Boolean powers, recursive models, and the Horn

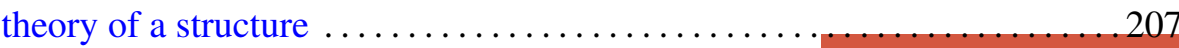

W. J. Phillips, Flow under a function and discrete decomposition of properly infinite $W^{*}$-algebras

Teodor C. Przymusiński, A solution to a problem of E. Michael ... 235

Bruce Harvey Wagner, Derivations of quasitriangular algebras 243 